\title{
Estimation of shear stress by using a myocardial bridge-mural coronary artery simulating device
}

\author{
Hao Ding ${ }^{1,2}$, Qian Yang ${ }^{2}$, Kun Shang ${ }^{1,2}$, Hailian $\mathrm{Lan}^{2}$, Jie Lv ${ }^{1,2}$, \\ Zhilin $\mathrm{Liu}^{3}$, Yang Liu ${ }^{1}$, Lixing Sheng ${ }^{1,2}$, Yanjun Zeng ${ }^{4}$ \\ ${ }^{1}$ Shanghai University of Medicine and Health Sciences, Shanghai, China \\ ${ }^{2}$ School of Medical Instrument and Food Engineering, \\ University of Shanghai for Science and Technology, Shanghai, China \\ ${ }^{3}$ Shanghai General Hospital, Shanghai, China \\ ${ }^{4}$ Biomechanics and Medical Information Institute, Beijing University of Technology, Beijing, China
}

\begin{abstract}
Background: This study was aimed at developing a myocardial bridge-mural coronary artery simulative device and analyzing the relationship between shear stress on the mural coronary artery and atherosclerosis.

Methods: A myocardial bridge-mural coronary artery simulative device was used to simulate experiments in vitro. In the condition of maintaining any related parameters such as system temperature, average flow rate, and heart rate, we calculated and observed changes in proximal and distal mean values, and oscillatory value of shear stress on the mural coronary artery by regulating the compression level of the myocardial bridge to the mural coronary artery.

Results: Under $0 \%$ compression, no significant differences were observed in distal and proximal mean values and oscillatory value of the shear stress on the mural coronary artery. With the increase in the degree of compression, the mean shear stress at the distal end was greater than that at the proximal end, but the oscillatory value of the shear stress at the proximal end was greater than that at the distal end. Conclusions: The experimental results of this study indicate that myocardial bridge compression leads to abnormal hemodynamics at the proximal end of the mural coronary artery. This abnormal phenomenon is of great significance in the study of atherosclerosis hemodynamic pathogenesis, which has potential clinical value for pathological effects and treatments of myocardial bridge. (Cardiol J 2017; 24, 5: 530-538)
\end{abstract}

Key words: mural coronary artery, myocardial bridge, hemodynamic, shear stress

\section{Introduction}

The coronary artery and its branches usually course above the surface of the myocardium through the epicardial fat or deep into the heart epicardium. Muscle overlaying the mural coronary (MC) artery is termed myocardial bridge (MB), and the segments of the coronary artery covered by the MB are called "mural coronary artery". MB is a common anatomical phenomenon, with an incidence between $15 \%$ and $85 \%$ [1-5]. In the normal anatomic position of the heart, the entrance of the $\mathrm{MC}$ is the proximal end and its exit is the distal end.

MB-MC artery is a complex condition with a unique anatomical variation. Several studies demonstrated that MB-MC artery is associated with cardiovascular diseases such as atherosclerosis and myocardial ischemia. Some researchers proposed

Address for correspondence: Dr. Yanjun Zeng, Biomechanics and Medical Information Institute, Beijing University of Technology, Beijing, China, tel: (8610) 67391809; fax: (8610) 67391975, e-mail: yjzeng@bjut.edu.cn 


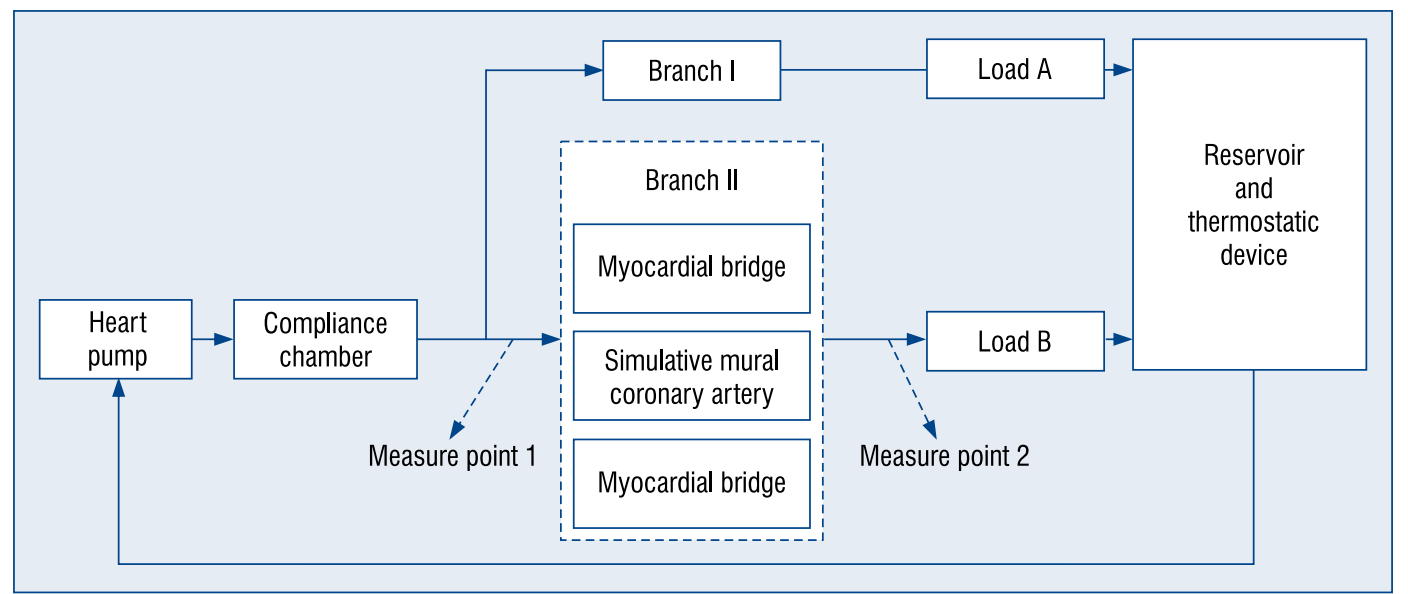

Figure 1. A block diagram of the myocardial bridge-mural coronary artery simulated device.

a high incidence of atherosclerosis at the proximal end of MC [6] but a low incidence of atherosclerosis formation in the $\mathrm{MC}$ segment and its distal end [7-11]. Atherosclerosis is closely related to the shear stress on blood vessels. Abnormal shear stress can alter the morphological structure and function of endothelial cells by activating multiple signal channels [12-15]. Pan [16] suggested that low shear stress and oscillatory shear stress can promote the formation of atherosclerosis by increasing the adhesion of leukocytes, plasma lipoprotein permeability, and the migration of smooth muscle cell and reactive oxygen species. Therefore, the study of abnormal shear stress can provide clinical reference for the investigation of the relationship between shear stress and atherosclerosis.

By using the MB-MC artery simulative device, which was designed by our research group, this study aimed at investigating the relationship between change in the shear stress on the coronary artery and high incidence of atherosclerosis at its proximal end under the circumstance of $\mathrm{MB}$ by simulating experiments in vitro and analyzing the corresponding shear stress at the proximal and distal ends of the MC.

\section{Methods}

\section{Structure and function of the device}

The MB-MC artery simulative device, as illustrated in Figure 1, is a circulatory system that includes a heart pump, compliance chamber, MB, simulative MC, afterload, reservoir, thermostatic device, and measuring system.

Two basic functions of the MB-MC artery simulative device are as follows: to simulate blood circulation, adjust, measure, provide real-time display, and record parameters of the blood circulatory system such as heart rate, velocity, pressure and temperature; and to provide a three-dimensional flow field and an adjustable shear stress environment for pipe flow chamber, and create an oppressive force on the MC artery.

The parameters of the simulative circulatory system can change within a certain scope. The parameters include heart rate, velocity, pressure, stroke volume, degree of pressure on the coronary artery, MB width, and the load in the coronary artery branches. The heart pump, which is used to simulate the heart, including its pressure range of 0 to $26.66 \mathrm{kPa}(0-200 \mathrm{~mm} \mathrm{Hg}, 1 \mathrm{~mm} \mathrm{Hg}=0.133$ $\mathrm{kPa}$ ), its flow rate range of 0.25 to $1.25 \mathrm{~L} / \mathrm{min}$, and its frequency of 40 to 200 times/min.

\section{Heart pump}

The study employed a scheme of mechanical simulation in which the design of the cam is a crucial issue. Our design basis is as follows: when the pump is running, the total resistance remains constant; thus, the relationship between output pressure $P(t)$ and output flow volume $Q(t)$ is linear. If the cross-sectional area of the piston pump is kept invariable, from the equation $Q(t)=V(t) A$ we observed that $P(t)$ and the piston velocity $V(t)$ also had a linear relationship. $A$ represents the cross-sectional area. Thus, if $P(t)$ is known, we can obtain the velocity curve of the piston. Therefore, the problem is transformed into solving the cam contour that drives the piston to motion based on the known piston velocity curve $V(t)$. This can be realized by integrating the velocity curve and appropriately dealing with the integral value. The 
results of the experiments show that the simulated pressure curve is relatively consistent with the pressure curve of the human heart [17].

\section{Compliance chamber}

The function of the compliance chamber is to simulate the elastic function of the aorta and change the intermittent blood ejection of the pulsatile pump into a continuous pulsatile flow. The compliance chamber can store and discharge energy for the system. The volume of the gas chamber can be calculated with the equation [18]:

$$
V_{o}=\frac{\Delta V}{P_{2}\left(\frac{1}{P_{2}}-\frac{1}{P_{1}}\right)}
$$

where $\Delta V$ is the pump output per pulse, and $P_{1}$ and $P_{2}$ are the systolic and diastolic pressures, respectively.

The total gas volume $V_{0}$ correspondingly varies with different $P_{1}$ and $P_{2}$ values. However, the following law is always satisfied: the smaller the difference between $P_{1}$ and $P_{2}$, the larger the $V_{0}$. When $P_{1}=P_{2}, V_{0}$ is infinite. In order to make the pulsatile amplitude adjustable within the stated range, the total compliance chamber volume $V_{0}$ should be between 200 and $400 \mathrm{~mL}$. Figure 2 shows a picture of the compliance chamber.

\section{$\mathrm{MB}$ and simulated MC module}

The simulated MC module is a closed Lucite cube, and the internal situation can be observed clearly. The internal size of the module is $125 \times$ $\times 110 \times 55 \mathrm{~mm}$ (length $\times$ width $\times$ height). The volume of the module is much larger than that of the simulated coronary artery. The device can simulate the coronary artery with an inner diameter of $4-5 \mathrm{~mm}$ and length of $80 \mathrm{~mm}$. Measuring point 1 is set at the inlet of the module to measure the proximal velocity, while measuring point 2 is located at the outlet to measure the distal velocity. The MB blocks are set at both radial sides of the simulated MC. The blocks, which are driven by a motor, undergo the straight reciprocating motion along the guide rail according to the preset depth to compress the simulated MC. The device can perform bilateral or unilateral compression, and different sized blocks can be chosen to get different compression width. The simulated MC is made of silicone tube, with an internal diameter of $4.33 \mathrm{~mm}$ and external diameter of $4.93 \mathrm{~mm}$. Figure 3 shows the MB and the simulated MC module structure.

The MB and simulated MC module can simulate two working cases (Fig. 4). Figure 4A shows

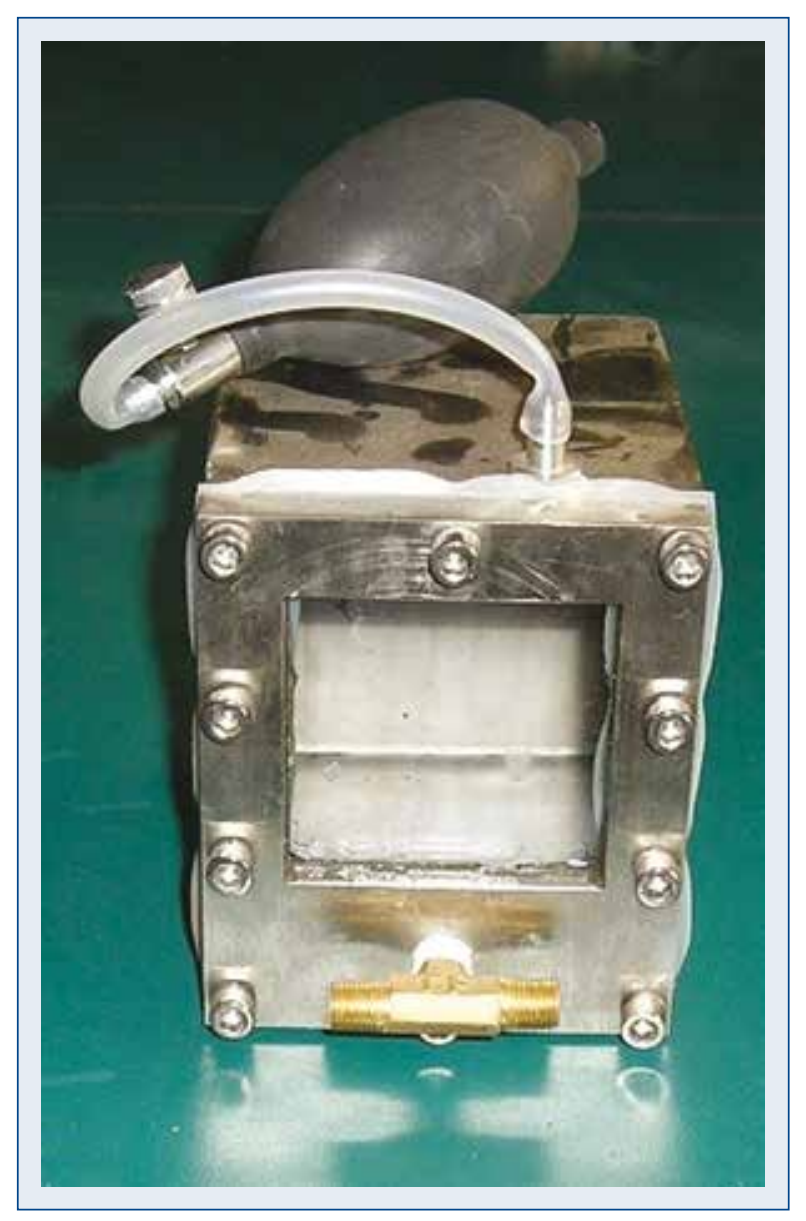

Figure 2. The compliance chamber.

the normal coronary artery without MB compression. Figure $4 \mathrm{~B}$ shows a case of systolic compression that reduced the size of the lumen area of the $\mathrm{MC}$, as the compression depth and width are adjustable.

\section{Afterload}

The function of the afterload is to simulate the peripheral resistance of the microvessels and capillary vessels (lumped resistance model). Regarding their physiological structure, the capillary vessels are very long and resistance is distributed along their total length without converging at one point. As far as capillary vessels themselves are concerned, the distribution of resistance is related to their diameters, lengths, and patterns of branches. Thus, in this case, a lumped resistance model does not work. However, when studying the blood flow in the large arteries far upstream from the capillary vessels, considering the influence of capillary vessels as "lumped resistance" is not only feasible but also technically convenient [19]. 


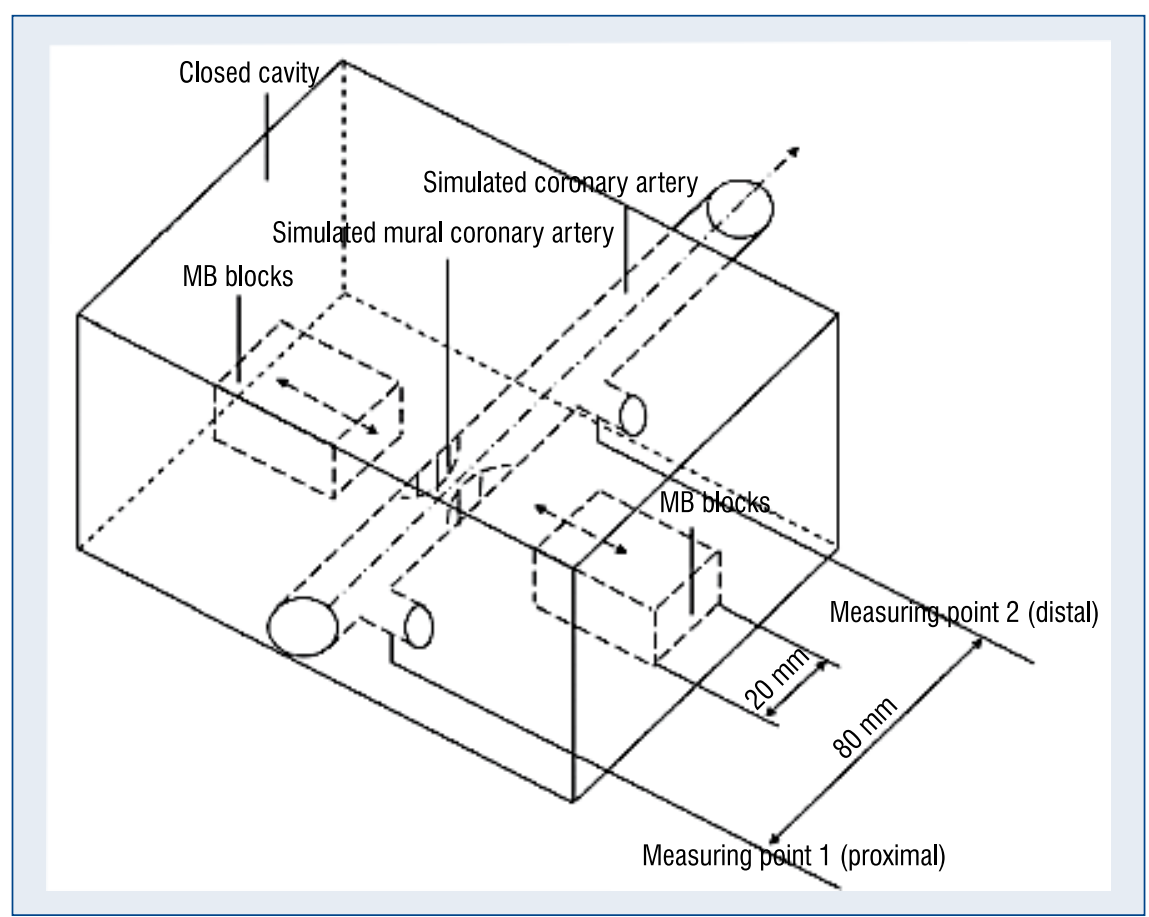

Figure 3. Myocardial bridge (MB) and simulated mural coronary module structure.

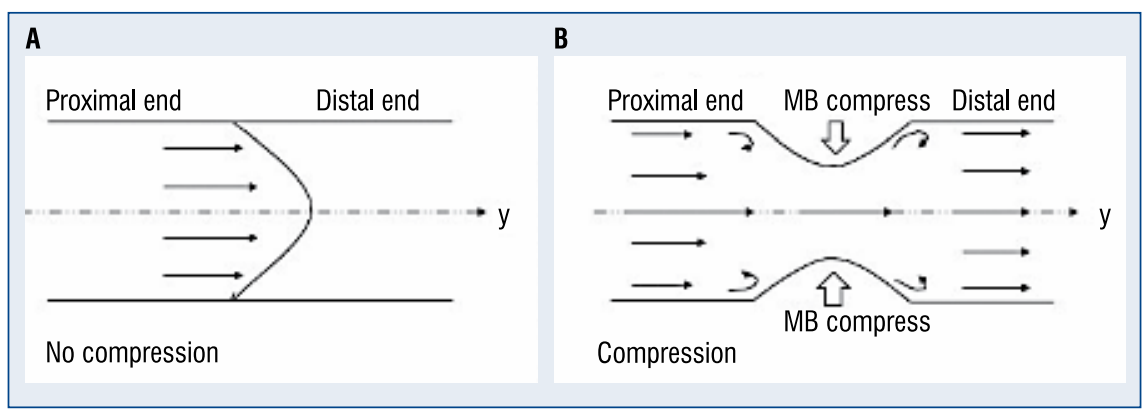

Figure 4. Myocardial bridge (MB) system.

\section{Reservoir and thermostatic device}

The reservoir stores liquid and maintains system circulation.

The thermostatic device maintains the normal physiological temperature of $37 \pm 1^{\circ} \mathrm{C}$.

\section{Measuring system}

The measuring system in the model is made up of existing meter instruments. Test points 1 and 2 are set at approximately $3 \mathrm{~cm}$ from the segments proximal and distal to MB. By connecting a Y-shaped tube, a Doppler flow wire (US Cardio-metrics FloMapll ${ }^{\circledast}$ Doppler velocity meter and 0.014-in Doppler guide wire of the Flo wire) can be inserted in the pipeline to measure and record the velocity where the ultrasonic detector is located.

\section{The degree of narrowing} of the MC artery diameter

Noble et al. [20] divided the degree of narrowing of the MC during systole into three grades as follows: grade $1(<50 \%)$, no clinical symptoms; grade 2 (50-75\% narrowing), increased lactic acid levels with myocardial ischemia; and grade 3 (> 75\% narrowing), significantly increased lactic acid, and presence of some clinical symptoms. In order to meet the needs in clinical research, the MB-MC artery simulative device can simulate the compression state of the above-mentioned three 
MB levels and can be used to calculate the shear stress at the proximal and distal ends of the MC.

\section{Theoretical foundation}

So far, the direct measurement of circular tube wall shear stress still has many technical difficulties, as it requires not only the detection of the velocity gradient near the circular tube wall with a relatively high spatial resolution but also ensuring that the circular tube wall is free of damage and has real-time continuous measurements. Xu et al. [21] and Liu et al. [22] proposed a method to calculate the shear stress on circular tube wall by measuring the flow rate of tube cross-section. By using this method, we calculated the wall shear stress at the proximal and distal ends of the simulative $\mathrm{MC}$ in the experimental device.

In our calculation, we assumed that the MC is isotropic and had a thin-walled linear elastomer and unchanged thickness under external force. The $\mathrm{MC}$ formed an axially symmetrical ring-like local narrow under MB compression (Fig. 4).

The blood flow velocity $(V[0, t])$ of the pipe cross-section $(y=0)$ at the proximal and distal ends of the simulative MC was calculated by using an ultrasonographic Doppler velocity meter, and the wall shear stress was calculated according to $V(0, t)$.

$\bar{\tau}(\mathrm{y})=\frac{\mu}{R} \mathrm{~V}_{0} \mathrm{y}$

$\tilde{\tau}(y, t)=\frac{\mu}{R} \cdot \operatorname{Re}\left\{\sum_{k=1}^{N} \frac{j^{3 / 2} a_{\mathrm{k}} J_{1}\left(j^{3 / 2} a_{\mathrm{k}} \cdot y\right)}{1-J_{0}\left(j^{3 / 2} a_{\mathrm{k}}\right)} V_{\mathrm{k}} \cdot e^{j k \omega t}\right\}$

$\tau(y, t)=\frac{\mu}{R} V_{0} y+\frac{\mu}{R} \cdot \operatorname{Re}\left\{\sum_{k=1}^{N} \frac{j^{3 / 2} a_{\mathrm{k}} J_{1}\left(j^{3 / 2} a_{\mathrm{k}} \cdot y\right)}{1-J_{0}\left(j^{3 / 2} a_{\mathrm{k}}\right)} V_{\mathrm{k}} \cdot e^{j k \omega t}\right\}$

The average shear stress (i.e., steady shear stress) $\bar{\tau}(y)$, pulsating shear stress $\tau(y, t)$ and the total periodic shear stress $\tau(y, t)$ were calculated according to equations (1), (2), and (3).

In the equations, $\mu$ is the dynamic viscosity of simulative blood; $\rho$ is the density of simulative blood; $R$ is the inner diameter of the simulative artery; $\mathrm{j}=\sqrt{-1} ; J_{0}$ is the zero-order Bessel function; $J_{1}$ is the first-order Bessel function;

$\omega=\frac{2 \pi}{T} ; \mathrm{y}=\frac{r}{R}$;

$\alpha_{\mathrm{k}}$ is the Womersley number; $k=0,1,2 \ldots \ldots \mathrm{N}$; $V_{0}$ is the zero-order velocity coefficient of pipe axial; VK is the K-order coefficient of pipe axial.

In the calculation, the related parameters of simulated $\mathrm{MC}$ are as follows: the inner radius
$\mathrm{R}=2.165 \times 10^{-3} \mathrm{~m}$, simulated blood density $\mathrm{r}=1.05 \times 103 \mathrm{~kg} / \mathrm{m}^{3}$, dynamic viscosity $\mathrm{m}=3.8 \times$ $\times 10^{-3} \mathrm{~Pa} \cdot \mathrm{s}$, and cardiac cycle $\mathrm{T}=0.85 \mathrm{~s}$.

\section{Experimental design}

The liquid in the circulatory system of the simulative device is a mixture of low-molecularweight dextran and normal saline in the ratio of $3: 1$ with a viscosity of $3.8 \times 10^{-3} \mathrm{~Pa} \cdot \mathrm{s}$ [23].

The specific experimental operation is as follows:

1. Start the heart pump motor, let the motor control the movement of the heart pump to simulate the human heart, and then let the circulatory system start running.

2. According to clinical normal human physiological parameters, preset the heart rate of the simulator at $70 \mathrm{bpm}$, adjust the accumulator and loads $\mathrm{A}$ and $\mathrm{B}$ to attain a systolic blood pressure of $120 \mathrm{~mm} \mathrm{Hg}$ of the simulative MC and a diastolic pressure of $80 \mathrm{~mm} \mathrm{Hg}$, and set the average flow rate to $250 \mathrm{~mL} / \mathrm{min}$. Meanwhile, keep the pressure waveform in stable shape during the movement.

3. Maintain the preset system temperature, the average flow rate, and heart rate unchanged during the experiment. Start the MB squeezing block machine. The squeezing block with a preset compression width creates a reciprocating and bidirectional compression on the pipe flow chamber according to the preset condition. The MB compresses the MC when the heart is systolic [24]. Thus, the frequency of the MB compression must be consistent with the heart systolic frequency. Besides, the maximum output pressure of the heart must keep pace with the maximum amount of compression of the MB.

4. Adjust the degree of the compression on the $\mathrm{MC}$, and repeat the experiment several times. Calculate and observe the changes in the proximal and distal mean value, and oscillatory value of the shear stress on the MC artery by using a computer when the degrees of compression are $0 \%, 50 \%$, and $80 \%$.

\section{Main outcome measures}

The simulated results of the shear stress pulse waveform; the proximal and distal shear stress under the compression of MB; and the adjustment results of shear stress. 

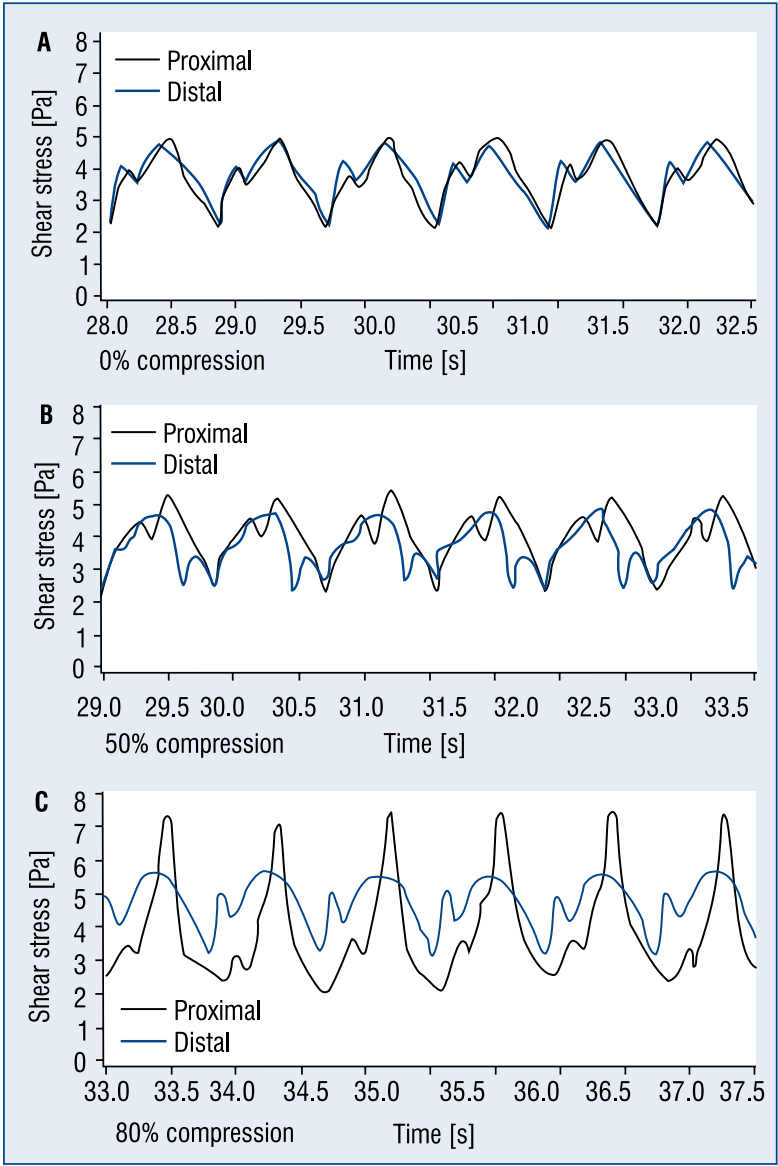

Figure 5. Waveforms of shear stress under $0 \%$ (A), 50\% (B), and $80 \%$ (C) of compression of the myocardial bridge to the mural coronary artery.

\section{Statistical analysis}

Data were processed by performing a variance analysis with the SPSS 17.0 statistical software. Data were expressed in the form of $X \pm S$.

\section{Results}

\section{The simulated results of shear} stress pulse waveform

In the simulated experiment, the pulse waveform of shear stress was observed under different degrees of compression and the shear stress at the proximal and distal ends of the $\mathrm{MC}$ was calculated. Figure 5 shows a waveform of shear stress under $0 \%, 50 \%$, and $80 \%$ compression of the MB to the MC artery.

As shown in Figure 5A, under $0 \%$ compression, the waveforms of shear stress at the proximal and distal ends of the $\mathrm{MC}$ were almost overlapping. Figure $5 \mathrm{~B}$ shows that under $50 \%$ compression, the shear stress amplitude at the proximal end was
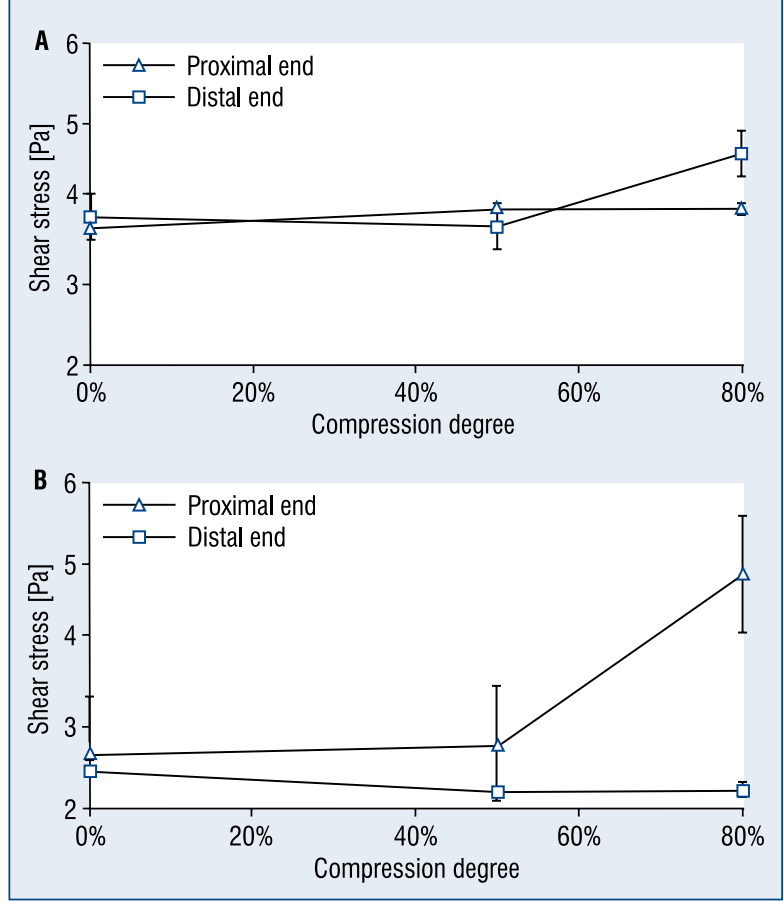

Figure 6. Mean (A) and oscillatory values (B) of the shear stress in the simulated mural coronary artery.

higher than that at the distal end. As illustrated in Figure 5C, under $80 \%$ compression, the difference in shear stress amplitude increased.

\section{The proximal and distal shear stress} under the MB compression

The proximal and distal shear stress values under different degrees of $\mathrm{MB}$ compression were calculated. A curve (Fig. 6A) for the mean value of shear stress in the simulated MC was drawn according to the experimental data. With the increase in the degree of compression, the mean shear stress at the proximal end had little change and the distal mean shear stress increased, with the distal mean shear stress being higher than the proximal mean shear stress. With the increase in the degree of compression, the shear stress on the simulative MC had violent shocks, and the degree of shocks at the proximal end was higher than that at the distal end (Fig. 6B).

Under $0 \%$ compression, the differences in mean and oscillatory values of shear stress at the proximal and distal ends were not significant. Under 50\% compression, the differences between the proximal oscillatory and distal oscillatory shear stress values were significant, and the oscillatory shear stress value was significantly greater at the proximal end than at the distal end. Under $80 \%$ compression, both the mean and oscillatory values of shear stress at the 
Table 1. Comparison of proximal and distal shear stress values of the simulated mural coronary artery under various degrees of compressions of the myocardial bridge $(x \pm s, n=15)$.

\begin{tabular}{lccc}
\hline & \multicolumn{3}{c}{ Degrees of compression } \\
\cline { 2 - 4 } & $0 \%$ & $50 \%$ & $80 \%$ \\
\hline Mean value $[\mathrm{Pa}]:$ & & & $3.949 \pm 0.075$ \\
$\quad$ Proximal shear stress & $3.705 \pm 0.052$ & $3.945 \pm 0.054$ & $4.628 \pm 0.038$ \\
$\quad$ Distal shear stress & $3.846 \pm 0.044$ & $3.719 \pm 0.045$ & $4.879 \pm 0.038$ \\
Oscillatory value [Pa]: & & & $2.218 \pm 0.538$ \\
$\quad$ Proximal shear stress & $2.636 \pm 0.040$ & $2.765 \pm 0.040$ & $2.164 \pm 0.042$ \\
$\quad$ Distal shear stress & $2.444 \pm 0.042$ & & \\
\hline
\end{tabular}

proximal and distal ends were all significantly different. The mean value of shear stress at the distal end was higher than that at the proximal end, and the oscillatory value of shear stress at the proximal end was higher than that at the distal end (Table 1).

\section{The adjustment results of shear stress}

In the simulative device, keep the system under constant temperature, flow rate, and heart rate. The redistribution of each branch flow can be realized by adjusting the afterload. Adjust afterloads A and B. The smaller afterload B and the greater afterload $\mathrm{A}$, the greater the shear stress. Vice versa, the shear stress is smaller.

\section{Discussion}

According to the simulation experiment in vitro, with the increase in the degree of $\mathrm{MB}$ compression, the distal mean shear stress is higher than the proximal mean shear stress. The reason for this phenomenon is that at the instance the MB compresses to the $\mathrm{MC}$ artery, the liquid flow rate at the MC distal end increases sharply, while the liquid flow at the proximal end is hindered and its flow rate is decreased.

Under $0 \%$ compression, both ends of the MC flow remained constant. The shear stress amplitude at the proximal end was consistent with that at the distal end. With the increase in compression degree, proximal and distal shear stress waveforms begin to change. This is because when the MB compresses the $\mathrm{MC}$ artery, the lumen area of the $\mathrm{MC}$ decreases, resulting in retardation of the blood flow, leading to a sharp decline in proximal flow rate and shear stress, and to a distal blood flow rate and increased shear stress. When the compression was released, the blood at the proximal and distal ends flowed into the $\mathrm{MC}$ to fill the vacuum in the $\mathrm{MC}$ segment. This resulted in sharp increases in flow rate and shear stress. Meanwhile the distal flow rate and shear stress decreased.

The experimental results show that with the increase in the degrees of compression, the oscillatory value of the shear stress at the proximal end was higher than that at the distal end, and the mean value of the shear stress at the proximal end was less than that at the distal end. If the mean value of shear stress determines the extent of endothelial cell injury, the proximal end should have a high incidence of atherosclerosis, which is inconsistent with the clinical facts [8-10]. Conversely, if the oscillatory value of shear stress determines the extent of endothelial cell injury, the distal end should have a high incidence of atherosclerosis. The increase in oscillatory shear stress at the proximal end caused the $\mathrm{MC}$ to be under a fatigue loading state for a long time, which will cause fatigue damage to endothelial cells and lead to coronary artery pathological changes, thereby causing related diseases such as atherosclerosis [25-36]. The simulated experiment results are consistent with the results of Ishii et al. [8] and Chiu et al. [9], i.e., the proximal end has a high incidence of atherosclerosis. Therefore, we conclude that the increase in oscillatory shear stress should be the main cause of endothelial cell injury. These conclusions have been verified by an experiment in another study [37].

The purpose of this study was to perform a simulation experiment in vitro along with a review of related literatures to present a reasonable mechanical explanation of the results of the experiment. We propose that the increase in the oscillatory value of shear stress at the proximal end is the main reason for the high incidence of atherosclerosis at the proximal end. This will provide 
references for studies on the relationship between shear stress and atherosclerosis.

Owing to the strong axial constraint of the connective tissue, the axial elastic deformation of the artery pipe is so slight that it can be ignored in the pipe axial velocity analysis [38]. Therefore, considering the artery pipe as a rigid pipe when analyzing the shear stress distribution in the artery pipe is a reasonable approximation. We calculated the Reynolds numbers for the simulated blood at the proximal and distal ends of the simulative MC and obtained a Reynolds number of $<2,000$ for both. It means that the flow at the proximal and distal ends of the simulative $\mathrm{MC}$ is laminar. In this study, the inner diameters of the deformations at the proximal and distal ends of the simulative $\mathrm{MC}$ were so small that they can be ignored. The abovementioned conditions agree with the applicable conditions for equations 1 to 3 . However, equations 1 to 3 should not be used for calculating the shear stress when the diameter of the MC is markedly changed. The shear stress calculation method needs to be further discussed in a future work.

\section{Limitation of the study}

In this study, the simulation experiment was based on the normal human physiological parameters. The device simulated a heart rate of 70 times/min, systolic blood pressure of $120 \mathrm{~mm} \mathrm{Hg}$, diastolic pressure of $80 \mathrm{~mm} \mathrm{Hg}$, and average flow rate of $250 \mathrm{~mL} / \mathrm{min}$.

Because of the limitation of the experimental device, we consider the coronary artery as a straight tube. The diameter of the tube is not changed. In fact, coronary arteries are not straight but bending with a varying radius during the heart cycle.

\section{Conclusions}

To simulate the changes in the hemodynamic parameters such as heart rate, flow rate, diameter of the tube coronary artery and shear stress in various situations, a comprehensive study on the relationship between these changes and atherosclerosis should be conducted in our subsequent research.

Funding: The work of this project was supported by "Chen Guang” program sponsored by Shanghai Municipal Education Commission and Shanghai Education Development Foundation (No. 13CGB09) and "Minimally Invasive Inspiring Innovation Fund" program sponsored by University of Shanghai for Science and Technology, School of Medical Instrument and Food Engineering (No.YS30808121) and Shanghai Natural Science Foundation Project sponsored by Science and Technology Commission of Shanghai Municipality (No. 17ZR1413500).

\section{Conflict of interest: None declared}

\section{References}

1. Balamuthusamy S, Kosla S, Benatar D, et al. Myocardial infarction in a young African-American male due to myocardial bridging. Cardiology. 2006; 105(3): 165-167, doi: 10.1159/000091400, indexed in Pubmed: 16479103.

2. Yetman AT, McCrindle BW, MacDonald C, et al. Myocardial bridging in children with hypertrophic cardiomyopathy--a risk factor for sudden death. N Engl J Med. 1998; 339(17): 1201-1209, doi: 10.1056/NEJM199810223391704, indexed in Pubmed: 9780340.

3. Channer KS, Bukis E, Hartnell G, et al. Myocardial bridging of the coronary arteries. Clin Radiol. 1989; 40(4): 355-359, indexed in Pubmed: 2527105.

4. Hansen BF. Myocardial covering on epicardial coronary arteries. Prevalence, localization and significance. Scand J Thorac Cardiovasc Surg. 1982; 16(2): 151-155, indexed in Pubmed: 7156925.

5. Debbarma J, Das P, Debbarma A, et al. Myocardial bridging and sudden death: a care report. J Evolution Med Dental Scien. 2002; 4: 116-119.

6. Alegria JR, Herrmann J, Holmes DR, et al. Myocardial bridging. Eur Heart J. 2005; 26(12): 1159-1168, doi: 10.1093/eurheartj/ /ehi203, indexed in Pubmed: 15764618.

7. Ishii T, Asuwa N, Masuda S, et al. The effects of a myocardial bridge on coronary atherosclerosis and ischaemia. J Pathol. 1998; 185(1): 4-9, doi: 10.1002/(SICI)10969896(199805)185:1<4::AID-PATH50>3.0.CO;2-3, indexed in Pubmed: 9713353.

8. Ishii T, Hosoda Y, Osaka T, et al. The significance of myocardial bridge upon atherosclerosis in the left anterior descending coronary artery. J Pathol. 1986; 148(4): 279-291, doi: 10.1002/ /path.1711480404, indexed in Pubmed: 3701494.

9. Chiu JJ, Wang DL, Chien S, et al. Effects of disturbed flow on endothelial cells. J Biomech Eng. 1998; 120(1): 2-8, indexed in Pubmed: 9675673.

10. Rosencrance G, Deer TR, Lee KC, et al. Coronary artery muscle bridging causing class III angina in a patient with no coronary atherosclerosis. W V Med J. 1995; 91(5): 196-197, indexed in Pubmed: 7660654.

11. Zheng Z, Xu G, Li W, et al. Effect of myocardial bridge on blood flow of coronary arter. J Shanghai Jiaotong University (Med Sci). 2006; 26: 166-168.

12. Warboys CM, Amini N, de Luca A, et al. The role of blood flow in determining the sites of atherosclerotic plaques. F1000 Med Rep. 2011; 3: 5, doi: 10.3410/M3-5, indexed in Pubmed: 21654925.

13. Woo KV, Qu X, Babaev VR, et al. Tie1 attenuation reduces murine atherosclerosis in a dose-dependent and shear stress-specific manner. J Clin Invest. 2011; 121(4): 1624-1635, doi: 10.1172/ /JCI42040, indexed in Pubmed: 21383501.

14. Ravensbergen J, Ravensbergen JW, Krijger JK, et al. Localizing role of hemodynamics in atherosclerosis in several human vertebrobasilar junction geometries. Arterioscler Thromb Vasc Biol. 1998; 18(5): 708-716, indexed in Pubmed: 9598828. 
15. Jenei C, Balogh E, Szabo GT, et al. Wall shear stress in the development of in-stress restenosis revised. A critical review of clinical data on shear stress after intracoronary stent implantation. Cardiol J. 2016; 23(4): 365-373, doi: 10.5603/CJ.a2016.0047.

16. Pan S. Molecular mechanisms responsible for the atheroprotective effects of laminar shear stress. Antioxid Redox Signal. 2009; 11(7): 1669-1682, doi: 10.1089/ars.2009.2487, indexed in Pubmed: 19309258.

17. Ding H, Shen L, Xu GL, et al. Heart pump system in heart-coronary artery-myocardial bridge circulation apparatus. J University of Shanghai for Science and Technology. 2003; 25: 201-204.

18. Zhang H, Huang Y. Hydraulic transmission. Chinese Machine Press, Beijing. ; 1991.

19. Ding H, Qiao A, Shen L, et al. Design of compliance chamber and after-load in apparatus for cultured endothelial cells subjected to stresses. Cell Biol Int. 2006; 30(5): 439-444, doi: 10.1016/j. cellbi.2006.02.003, indexed in Pubmed: 16616864 .

20. Noble J, Bourassa MG, Dyrda I, et al. Myocardial bridging and milking effect of the left anterior descending coronary artery: normal variant or obstruction? Am J Cardiol. 1976; 37(7): 993-999, doi: 10.1016/0002-9149(76)90414-8, indexed in Pubmed: 1274883.

21. Xu G, Qin K, Liu Z, et al. Calculation of the shear stress in the parallel-plate flow chamber under pulsatile flow condition. Chinese Quarterly Mech. 2000; 21: 45-51.

22. Liu B, Liu Z. Fluid shear stress distribution of periodic oscillatory blood flow in rigid circular tube. Chinese Quarterly Mech. 2002; 23: 7-8.

23. Jun Y, Tao G. The discussion of normal blood viscosity. Chin J Hemorheol. 1998; 8: 69.

24. Zhang G, Ge J, Shen L, et al. Design and manufacture of medeling of fluid dynamics related to the myocardial bridging and mural coronary artery. J Biomed Eng. 2005; 22: 593-597.

25. Xinghua G, Hua W, Keqiang W, et al. The effect of shear stress on endothelium membrane fluidity. Chin J Anat. 2000; 23: 352-354.

26. Werner M, Bräunlich S, Ulrich M, et al. Drug-eluting stents for the treatment of vertebral artery origin stenosis. J Endovasc Ther. 2010; 17(2): 232-240, doi: 10.1583/09-2904.1, indexed in Pubmed: 20426646.

27. Haiqiang $Q$, Xingquan $Z$, Ping $Z$, et al. Preliminary study for association of blood pressure and heart rate on carotid hemodynamics. Chin J Stroke. 2012; 7: 461-465.
28. Niu J, Dai X, Qiao A, et al. Hemodynamics analysis of vertebral artery ostium stenosis treated with stent implantation undergoing different protrusion distances. J Med Biomech. 2012; 27: 598-602.

29. Gao J, Wang G, Xiao J. Numerical simulation of hemodynamics in suturing and sutureless anastomotic models. Chin J Biomed Engineering. 2012; 31: 866-874.

30. Mintz GS. Clinical utility of intravascular imaging and physiology in coronary artery disease. J Am Coll Cardiol. 2014; 64(2): 207-222, doi: 10.1016/j.jacc.2014.01.015, indexed in Pubmed: 24530669.

31. Peng H, Yang D. [A boundary element analysis on hemodynamic characteristics at the bifurcation of abdominal arterial]. Sheng Wu Yi Xue Gong Cheng Xue Za Zhi. 2012; 29(4): 697-700, indexed in Pubmed: 23016419.

32. Yang J, Liu J, Yu H, et al. Comparison of hemodynamics in iliac aortic with thrombosis and normal iliac aortic based on numerical simulation. BME Clin Med. 2012; 16: 1-6.

33. Ling S-L, Zhang Y, Liao B, et al. The influence of low laminar shear stress on the expression of scavenger receptor B I in endothelial cells. Chin J Arterioscler. 2009; 17: 661-664.

34. Stone PH, Coskun AU, Kinlay S, et al. Effect of endothelial shear stress on the progression of coronary artery disease, vas cular remodeling, and in-stent restenosis in humans: in vivo 6-month follow-up study. Circulation. 2003; 108(4): 438-444, doi: 10.1161/01.CIR.0000080882.35274.AD, indexed in Pubmed: 12860915.

35. Pivkin IV, Richardson PD, Laidlaw DH, et al. Combined effects of pulsatile flow and dynamic curvature on wall shear stress in a coronary artery bifurcation model. J Biomech. 2005; 38(6): 1283-1290, doi: 10.1016/j.jbiomech.2004.06.015, indexed in Pubmed: 15863113.

36. Chaichana T, Sun Z, Jewkes J. Impact of plaques in the left coronary artery on wall shear stress and pressure gradient in coronary side branches. Com Methods in Biomech Biomed Engineer. 2013; 17: 108-118.

37. Zhou Y, Ding H, Xu M, et al. Effect of oscillatory shear stress on endothelial cells in cell culture system. J Clin Rehab Tissue Eng Res. 2009; 13: 1669-1671.

38. Liu Z, Li X. Principles and methods of blood dynamics. Shanghai, Fudan University Press, 1997. 